\title{
Energia ultrassônica na avaliação da macroagregação de um Argissolo sob manejo conservacionista com diferentes plantas de cobertura
}

\section{Ultrasonic energy in the evaluation of macroaggregation of a Paleudalf under conservation management with different cover plants}

\author{
Érika Andressa da Silva \\ Universidade Estadual de Minas Gerais (UEMG) \\ E-mail: andressaerikasilva@gmail.com \\ OrcID: https://orcid.org/0000-0002-4718-2716 \\ Micael Stolben Mallmann \\ Universidade Federal de Santa Maria (UFSM) \\ E-mail: micaelstolben@gmail.com \\ OrclD: : https://orcid.org/0000-0003-0179-6031
}

Monike Andrade Pereira

Universidade Federal de Santa Maria (UFSM)

E-mail: monikeandrade.pereira@hotmail.com

OrcID: https://orcid.org/0000-0001-9890-2579

Dalvan José Reinert

Universidade Federal de Santa Maria (UFSM)

E-mail: dalvan@ufsm.br

OrcID: https://orcid.org/0000-0002-4671-8486

José Miguel Reichert Universidade Federal de Santa Maria (UFSM)

E-mail: reichert@ufsm.br

OrcID: https://orcid.org/0000-0001-9943-2898

Resumo: Sonificação tem sido uma ferramenta útil para mensurar a energia necessária para promover a dispersão dos agregados. Este trabalho objetivou avaliar o efeito de plantas de cobertura na macroagregação de um ARGISSOLO VERMELHO Distrófico arênico usando energia ultrassônica. Agregados (8-4,76 mm) foram coletados da camada de 0,0-0,05 m de profundidade em diferentes manejos com plantas de cobertura: VE - vegetação espontânea, AVNA - aveia preta + nabo forrageiro, AVER - aveia preta + ervilhaca peluda, GR - Grama Pensacola, AF - amendoim forrageiro. Solo descoberto (SD) foi utilizado como testemunha. Amostras compostas por $10 \mathrm{~g}$ de agregados foram submetidas à sonificação em níveis crescentes de energia. O uso do solo com VE afetou negativamente a estabilidade dos agregados, enquanto as plantas de cobertura AF, AVER e AVNA apresentaram resultados intermediários, com tendência a melhorar a agregação do solo em longo prazo. A macroagregação foi favorecida pelo manejo com GR, exigindo maior energia (> $33,5 \mathrm{~J} \mathrm{~mL}^{-1}$ ) para a dispersão do solo.

Palavras-chave: Índice de dispersão normalizado. Plantas de cobertura. Sonificação.

Abstract: Sonication has been a useful tool to evaluate the energy necessary to promote the dispersion of the soil aggregates. Our goal was to evaluate the effect of cover crops on the macroaggregation of a ARGISSOLO VERMELHO Distrófico arênico (Paleudalf) by ultrasonic energy. Aggregates were sampled at 0.0-0.05 m depth layer in five different managements with cover plants: SV - spontaneous vegetation, OT - black oat + forage turnips, OV - black oat + hairy vetch, PG - Pensacola grass, P - forage peanut. Bare soil (BS) was used as a control. The samples has a $10 \mathrm{~g}$ of soil aggregates were submitted to sonification with increasing energy levels. The soil use with SV showed negatively affects in the aggregates stability, while cover crops $\mathrm{P}$, OT and OV occurred intermediate results, with a tendency to improve soil aggregation long-term. 
Macroaggregation was influenced by soil management with PG, requiring greater energy $\left(>33.5 \mathrm{~J} \mathrm{~mL}^{-1}\right)$ for soil dispersion.

Keywords: Cover crops. Normalized dispersion index. Sonification.

Data de recebimento: 06/03/2021

Data de aprovação: 15/06/2021

\section{Introdução}

DOI: https://doi.org/10.30612/agrarian.v14i52.14311

Os sistemas de manejo do solo com plantas de cobertura incrementam a quantidade de fitomassa depositada na superfície do solo. A qualidade do material vegetal das plantas de cobertura exerce forte influência na agregação do solo (Loss, Pereira, Anjos, Giacomo, Perin, 2011; Tivet et al., 2013; Zhu, Angers, Field, Minasny, 2017; Silva et al., 2020). A ervilhaca e nabo forrageiro são leguminosas que apresentam compartimento lábil superior em aproximadamente duas vezes a gramínea aveia preta (Acosta, Amado, Silva, Santi, Weber, 2014). Devido a maior recalcitrância, os resíduos dessa gramínea permanecem por longo tempo sobre a superfície do solo (Aita \& Giacomini, 2003; Redin, Giacomini, Ferreira, Eckhardt, 2016). Deste modo, atua impedindo a ação direta das gotas de chuva. Além disso, a cobertura morta da aveia preta reduz a amplitude térmica e contribui para a manutenção da umidade no solo.

Ademais as gramíneas perenes apresentam um denso sistema radicular fasciculado que é renovado regularmente (Torres, Pereira, Assis, Souza, 2015). Associada ao aporte de carbono originado das raízes mortas, a liberação de exsudatos na rizosfera das raízes novas e finas estimula a atividade biológica conduzindo a formação e estabilização dos agregados do solo (Silva et al., 2016; Silva et al., 2019; Canalli et al., 2020).

Do ponto de vista de agricultura conservacionista, em relação ao monocultivo, o manejo com associações de plantas de cobertura, leguminosas e gramíneas, traz mais benefícios à agregação do solo (Silva et al., 2019). A diversidade vegetal potencializa a formação de macroagregados durante o desenvolvimento das culturas pela complexidade das relações ecológicas entre os sistemas radiculares de diferentes espécies vegetais (Loss et al., 2011; Loss et al., 2015).

Os macroagregados compreendem estruturas complexas e diversificadas, com grande capacidade para reter energia e matéria adicionada na forma de carbono (Briedis et al., 2012; Tivet et al., 2013). Em certas condições de manejo, a baixa estabilidade destes agregados reflete em processos erosivos no solo (Briedis et al., 2012), uma vez que a fragmentação em unidades menores (microagregados) pode proporcionar o entupimento de poros e selamento do solo, culminando em aumento das taxas de escoamento superficial (Stefanoski, Santos, Marchão, Petter, Pacheco, 2013; Tivet et al., 2013; Silva et al., 2019).

Ainda existem muitas lacunas no que diz respeito à escolha dos métodos de avaliação da estabilidade dos macroagregados grandes (8,0-4,76mm) (Lehtinen et al., 2014; Inagaki et al., 2016; Silva et al., 2016). Atualmente, estudos têm apontado que as avaliações de estabilidade desses agregados por técnicas de sonificação (ultrassonificação) são mais eficientes para distinguir os efeitos do uso do solo em sua dinâmica de agregação (Schomakers, Mentler, Steurer, Klik, Mayer, 2011; Lehtinen et al., 2014; Silva et al., 2016; Silva et al., 2019).

Em contraste com a maioria dos métodos convencionais, como peneiramento úmido, as técnicas ultrassônicas podem regular e quantificar o nível de energia aplicado aos agregados do solo (Ribeiro, Lima, Curi, Oliveira, 2013; Silva et al., 2019). Portanto, este estudo teve como objetivo avaliar os efeitos de diferentes coberturas vegetais na estabilização de macroagregados de um Argissolo utilizando energia ultrassônica.

\section{Material e Métodos}

\subsection{Descrição da área experimental}

O estudo foi conduzido na área experimental pertencente ao departamento de solos da Universidade Federal de Santa Maria (UFSM), Santa Maria, Rio Grande do Sul. O clima da região é o Cfa segundo a classificação de Köppen, o qual corresponde ao clima subtropical úmido, sem estiagens, com temperatura 
média do mês mais quente superior a $22^{\circ} \mathrm{C}$, e a temperatura do mês mais frio entre $-3^{\circ} \mathrm{C}$ e $18^{\circ} \mathrm{C}$ (Alvares, Stape, Sentelhas, Moraes, Sparovek, 2013).

O solo da área de estudo foi classificado como ARGISSOLO VERMELHO Distrófico arênico (Santos et al., 2018), e apresenta na camada de 0,0-0,05 m $654 \mathrm{~g} \mathrm{~kg}^{-1} ; 242 \mathrm{~g} \mathrm{~kg}^{-1}$ e $104 \mathrm{~g} \mathrm{~kg}^{-1}$ de areia, silte e argila, respectivamente. Em abril de 2010, no local do estudo foi conduzido um experimento para avaliar o efeito da injeção de dejeto líquido de suínos no solo. A área vinha sendo cultivada com sucessão aveia - milho até março de 2015, e a partir desta data a área permaneceu em pousio até fevereiro de 2016.

O experimento deste estudo foi implantado em abril de 2016. Os tratamentos consistiram na implantação de diferentes plantas de cobertura em monocultivo ou em sistemas de rotação. As plantas de cobertura utilizadas foram: Vegetação espontânea (VE); Aveia preta (Avena strigosa Schreb) + nabo forrageiro (Raphanus sativus L.) + Feijão miúdo (AVNA); Aveia preta (Avena strigosa Schreb) + ervilhaca peluda (Vicia villosa Roth) + feijão miúdo (AVER); Grama Pensacola - GR (Paspalum notatum), Amendoim forrageiro (Arachis pintoi) e uma testemunha para o ensaio, solo descoberto (SD).

As culturas de aveia + nabo e aveia + ervilhaca foram semeadas a lanço no período de outono inverno. Para as culturas de aveia e nabo utilizaram-se as porcentagens de $30 \%$ e $70 \%$ do total recomendado por hectare, sendo 100 e $15 \mathrm{~kg} \mathrm{ha}^{-1}$ respectivamente. As culturas de aveia e ervilhaca foram semeadas com porcentagens de $45 \%$ e $55 \%$ do total recomendado por hectare, sendo 100 e $80 \mathrm{~kg} \mathrm{ha}^{-1}$, respectivamente. Após 30 dias da emergência foi realizada adubação nitrogenada com $100 \mathrm{~kg}$ de ureia (42\% de N).

Antes da implantação das culturas realizou-se dessecação das parcelas, onde utilizou-se uma dose de $4 \mathrm{~L} \mathrm{ha}^{-1}$ de glifosato. No tratamento solo descoberto foram realizadas dessecações esporadicamente e no tratamento vegetação espontânea manteve-se a mesma composta principalmente por buva (Conyza bonariensis), grama (Paspalum notatum), aveia (Avena sativa), azevém (Lolium multiflorium), picão preto (Bidens pilosa) e serralha (Sonchus asper).

\subsection{Amostragem}

As amostras foram coletadas em Agosto de 2017. Para a avaliação da estabilidade dos agregados pela metodologia de sonificação, foram coletados monólitos de solo com auxílio de pá de corte nas camadas de 0,0-0,05 $\mathrm{m}$ de todos os tratamentos. As amostras indeformadas foram levadas para laboratórios e secas ao ar e posteriormente submetidas à separação manual dos agregados (por meio dos planos de fraqueza). Em seguida foram passados por um conjunto de peneiras de diâmetro de malha de 8,0 e 4,76 $\mathrm{mm}$, sendo selecionados os agregados retidos neste intervalo.

Ressalta-se que foi adotada a classificação para macroagregados descrita em Inagaki et al.(2016), em que macroagregados pequenos são aqueles com tamanhos variando de 0,25-2 mm de diâmetro, enquanto que macroagregados grandes tem tamanho variando 2-8 $\mathrm{mm}$ de diâmetro e os extragrandes o tamanho entre 8-19 mm de diâmetro. Desta forma, para a condução desta pesquisa foram selecionados macroagregados grandes de tamanho variando entre $8,0-4,76 \mathrm{~mm}$.

Seguindo a metodologia de Tedesco, Gianello, Bissani, Bohnen e Volkweiss (1995) foram realizadas análises para a caracterização dos atributos químicos do solo (Tabela 1). O carbono orgânico do solo (COS) foi determinado pelo método da combustão seca, por meio do analisador elementar, em que as amostras foram moídas em gral de ágata e acondicionadas em enpendorf de $2 \mathrm{ml}$ (Empresa Brasileira de Pesquisa Agropecuária [Embrapa], 2011).

Tabela 1. Caracterização química do ARGISSOLO VERMELHO Distrófico arênico sob manejo com diferentes plantas de cobertura.

\begin{tabular}{ccccccccc}
\hline Tratamentos & $\mathrm{pH}$ & $\mathrm{P}$ & $\mathrm{K}^{+}$ & $\mathrm{Ca}^{2+}$ & $\mathrm{Mg}^{2+}$ & $\mathrm{Al}^{3+}$ & $\mathrm{H}+\mathrm{Al}$ & $\mathrm{MOS}$ \\
\hline & & $\ldots \mathrm{mg} \mathrm{dm}^{-3} .$. & & $\ldots \ldots \ldots \ldots \ldots . \mathrm{cmol}_{c} \mathrm{dm}^{-3}$ & $\ldots \ldots \ldots \ldots \ldots$. & $\ldots .$. \\
AVER & 4,3 & 50 & 0,36 & 2,30 & 0,85 & 0,7 & 6,0 & 21 \\
AVNA & 4,3 & 46 & 0,34 & 1,92 & 0,64 & 0,75 & 6,4 & 23 \\
GR & 4,6 & 57 & 0,32 & 2,44 & 0,71 & 0,42 & 5,0 & 24 \\
SD & 4,2 & 77 & 0,20 & 1,52 & 0,53 & 1,1 & 6,4 & 21 \\
VE & 4,5 & 47 & 0,32 & 2,18 & 0,75 & 0,65 & 6,1 & 19 \\
AF & 4,6 & 36 & 0,16 & 2,35 & 0,88 & 0,52 & 6,0 & 17 \\
\hline
\end{tabular}

AVER: aveia + ervilhaca, AVNA: aveia + nabo, GR: Grama Pensacola, SD: solo descoberto, VE: vegetação espontânea, AF: amendoim Forrageiro, MOS: matéria orgânica do solo. 


\subsection{Avaliação da estabilidade de agregados por índices de dispersão}

Amostras compostas por $10 \mathrm{~g}$ de agregados da classe de tamanho 8-4,76 $\mathrm{mm}$ (base seca em estufa) foram transferidas para béqueres de $200 \mathrm{~mL}$ e com auxílio de uma bureta foram submersos em água destilada a partir de um gotejamento direcionado para a parede do béquer (que se encontrava com uma inclinação de $45^{\circ}$ ) numa taxa de 45 gotas por minuto. Quando toda massa de agregados estava submersa completou-se o volume do béquer e direcionaram-se as amostras para os ensaios de sonificação (Silva et al., 2016). Foram realizadas sonificações em aparelho da marca Vibracell Sonics, dotado de sonda de titânio de diâmetro 19,5 mm imersa a $1 \mathrm{~cm}$ na suspensão. Todas as amostras foram sonificadas em $40 \mu \mathrm{m}$ de amplitude e potencia de saída de $74,5 \mathrm{~W}$. Durante o processo de sonificação, cada amostra permaneceu em banho de gelo, mantendo a temperatura abaixo de $40^{\circ} \mathrm{C}$, conforme recomendado em trabalho com energia ultrassônica (Silva et al., 2016). A potência foi calibrada por técnicas calorimétricas (Ribeiro et al., 2017), conforme equação 1 :

$$
P=\left[\left(\left(m_{a} \cdot c_{a}\right)+c_{g}\right) \frac{\Delta T}{\Delta t}\right]
$$

Eq. 1

Onde: $\mathrm{P}$ é a potência determinada por calorimetria $(\mathrm{W})$; ma é a massa de água $(200 \mathrm{~g})$; ca é a capacidade de calor específico da água [4.186 $\left(\mathrm{g}^{\circ} \mathrm{C}^{-1}\right]$; $\mathrm{cg}$ é a capacidade de calor específica do béquer $(\mathrm{J}$ ${ }^{\circ} \mathrm{C}^{-1}$ ); $\Delta \mathrm{T}$ é o aumento da temperatura da água durante o período de tempo $\Delta \mathrm{t}$.

A capacidade de calor específica do béquer $(\mathrm{cg})$ foi calculada pela equação 2 :

$$
c_{g}=c_{v} \cdot m_{b}
$$

Eq.2

Onde: $\mathrm{cg}$ é a capacidade de calor específico do béquer $\left(\mathrm{J}^{\circ} \mathrm{C}^{-1}\right) ; \mathrm{cv}$ é a capacidade de calor específico do vidro $\left(840 \mathrm{~J}^{\circ} \mathrm{C}^{-1} \mathrm{~kg}^{-1}\right)$; e mb é a massa do béquer $(\mathrm{kg})$.

As amostras foram submetidas a tempos crescentes de sonificação durante 15, 45, 60 e 90 segundos. Com base nestes tempos empregados e na potência de funcionamento, os valores de energia ultrassônica específicos foram calculados pela equação 3 :

$$
E U=P \times T N
$$

Em que: EU é a energia ultrassônica aplicada à amostra $\left(\mathrm{J} \mathrm{mL}^{-1}\right)$; $\mathrm{P}$ é a potência $(\mathrm{W})$ emitida pelo aparelho e calculada através de calibração; T é o tempo de sonificação (s); $V$ é o volume da suspensão ( $\mathrm{mL}$ ). Desta forma cada tempo de sonificação corresponde, respectivamente, às energias específicas aplicadas: 0 ; 5,$58 ; 16,7 ; 22,3 ; 33,5 \mathrm{~J} \mathrm{~mL}^{-1}$.

Após cada sonificação, as amostras foram passadas em peneira de malha de $0,053 \mathrm{~mm}$. O material retido nessa peneira foi recolhido em latas de alumínio e conduzido a estufa para determinação da massa seca $\left(24\right.$ horas à $105^{\circ} \mathrm{C}$ ). A massa de agregados seca retida na peneira de $0,053 \mathrm{~mm}$ foi descontada da massa seca inicial de agregados conduzidos a sonificação, sendo que esta era composta por $10 \mathrm{~g}$ (base seca em estufa). Assim, por esta diferença obteve-se as massas das frações minerais (silte + argila) que passaram pela peneira de malha de $0,053 \mathrm{~mm}$. Os índices de dispersão (ID) foram calculados dividindo-se os teores de silte + argila pela amostra inicial. Os dados dos índices de dispersão, conforme sugerido por Sá, Lima, Silva e Dias Júnior (1999) foram normalizados para assim representarem apenas o efeito da estabilidade de agregados.

Os índices de dispersão normalizados (IDN) foram obtidos pela divisão de cada ID pelo máximo ID observado. Em seguida, foram construídas curvas de dispersão, plotando-se no eixo das abscissas a energia aplicada, em $\mathrm{J} \mathrm{mL}^{-1}$, e no eixo das ordenadas, os índices de dispersão normalizados $\left(\mathrm{g} \mathrm{g}^{-1}\right)$. Aos dados experimentais foram ajustados modelos hiperbólicos do tipo $\mathrm{Y}=\mathrm{X} /(\mathrm{a}+\mathrm{bX})$.

\subsection{Análises estatísticas}

Em campo, o experimento foi conduzido em blocos casualizados $6 \times 4$, sendo 6 tratamentos e 4 repetições (Blocos), onde cada parcela experimental continha $43 \mathrm{~m}^{2}$ de área útil $(3 \mathrm{~m} \times 14,5 \mathrm{~m})$. Em 
laboratório, os tratamentos foram comparados por cinco níveis de energia ultrassônica, totalizando 120 amostras (arranjo fatorial 6x4×5). Dentro de cada nível de energia, visando comparar os índices de dispersão normalizados encontrados para cada cobertura vegetal, com auxílio do software R (R Core Team, 2018) foi feita análise de variância e aplicado o teste de médias de Tukey $(p<0,05)$.

\section{Resultados}

Em todas as faixas de energia ultrassônica específica, o solo sob manejo com GR apresentou os agregados mais estáveis, o que foi sinalizado pelos menores índices de dispersão normalizados (Figura 1).

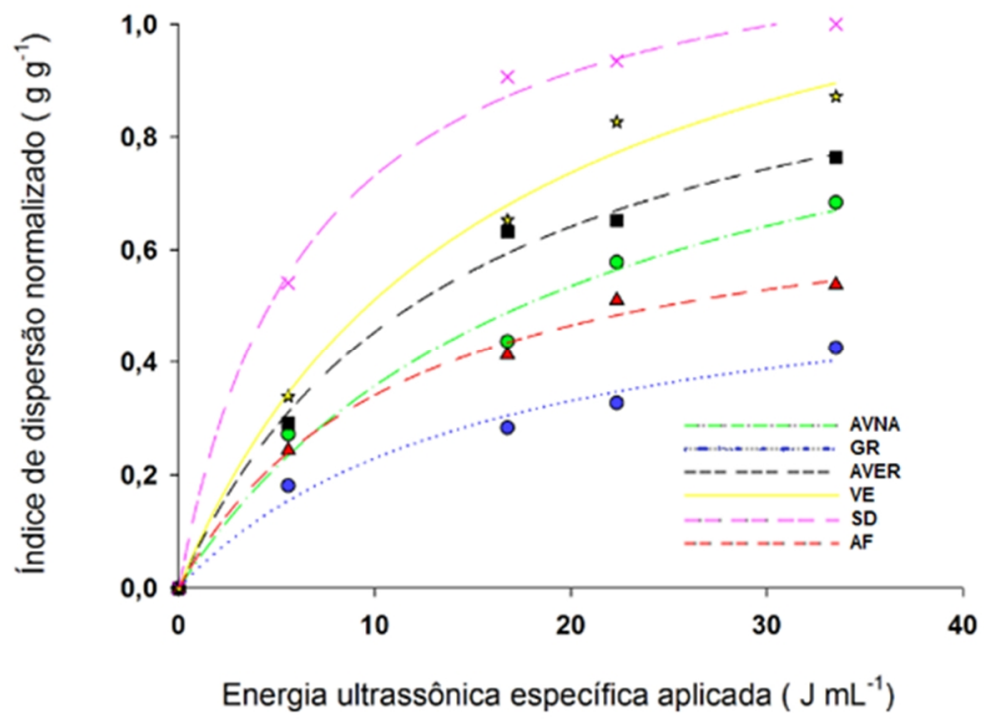

Figura 1. Curvas de dispersão do ARGISSOLO VERMELHO Distrófico arênico sob manejo com diferentes plantas de cobertura. AF: amendoim forrageiro, AVER: aveia + ervilhaca, AVNA: aveia + nabo, GR: grama Pensacola, SD: solo descoberto, VE: vegetação espontânea.

Tabela 2. Equações ajustadas para as curvas dos índices de dispersão normalizados (IDN) e teores de carbono orgânico do solo (COS) e cálcio trocável $\left(\mathrm{Ca}^{2+}\right)$ do ARGISSOLO VERMELHO Distrófico arênico sob diferentes manejos com plantas de cobertura.

\begin{tabular}{|c|c|c|c|c|c|}
\hline Uso Solo & Modelo ajustado & $\mathrm{R}^{2}$ & P-valor & $\begin{array}{c}\mathrm{COS} \\
\left(\mathrm{g} \mathrm{kg}^{-1}\right) \\
\end{array}$ & $\begin{array}{c}\mathrm{Ca}^{2+} \\
\left(\mathrm{cmol}_{\mathrm{c}} \mathrm{dm}^{-3}\right)\end{array}$ \\
\hline AVER & $\mathrm{IDN}=\mathrm{EU} /[12,8583+0,9157 \mathrm{EU}]$ & 0,99 & $p<0,0002$ & $10,3 a b$ & $2,30 \mathrm{a}$ \\
\hline AVNA & IDN = EU/[18,5895 + 0,9368EU] & 0,97 & $p<0,02$ & $10,2 a b$ & $1,92 \mathrm{ab}$ \\
\hline GR & IDN = EU/[26,7617 + 1,6848EU] & 0,95 & $p<0,001$ & 11,8 a & $2,44 \mathrm{a}$ \\
\hline SD & $\mathrm{IDN}=\mathrm{EU} /[5,4460+0,8212 \mathrm{EU}]$ & 0,99 & $p<0,0001$ & $8,6 \mathrm{~b}$ & $1,52 \mathrm{~b}$ \\
\hline VE & IDN= EU/[11,9405 + 0,7598EU] & 0,99 & $p<0,0003$ & $9,7 a b$ & $2,18 a b$ \\
\hline $\mathrm{AF}$ & IDN = EU/[15,6476 + 1,3652EU] & 0,96 & $p<0,006$ & $8,4 \mathrm{~b}$ & $2,35 \mathrm{a}$ \\
\hline
\end{tabular}

AVER: aveia + ervilhaca, AVNA: aveia + nabo, GR: Grama Pensacola, SD: solo descoberto, VE: vegetação espontânea, AF: amendoim Forrageiro. Médias seguidas de mesma letra na coluna não diferem entre si pelo teste de Tukey $(p<0,05)$.

Os manejos com AF apresentaram valores de COS semelhantes aos do SD (Tabela 2), porém, apresentaram menores índices de dispersão normalizados (Tabela 3).

A energia específica aplicada de $33,5 \mathrm{~J} \mathrm{~mL}^{-1}$ deixou o solo descoberto completamente disperso. $\mathrm{O}$ segundo nível de energia ultrassônica específica aplicado $\left(16,7 \mathrm{~J} \mathrm{~mL}^{-1}\right)$ discriminou os tratamentos em quatro grupos de estabilidade, nos quais o índice de dispersão normalizado diminuiu na seguinte ordem: $\mathrm{SD}>\mathrm{VE}=$ AVER > AVNA = AF > GR (Tabela 3).

Com a aplicação de $22,3 \mathrm{~J} \mathrm{~mL}^{-1}$, SD e VE apresentaram os maiores valores de IDN. O solo sob os manejos AVNA e AF apresentaram maior estabilidade estrutural que o solo manejado com VE e a testemunha SD, com menores índices de dispersão normalizados nos níveis de energia aplicados de 16,7 $\mathrm{JmL}^{-1} ; 22,3 \mathrm{JmL}^{-1}$ e 33,5 $\mathrm{JmL}^{-1}$ (Tabela 3). 
Tabela 3. Índices de dispersão normalizados do ARGISSOLO VERMELHO Distrófico arênico sob diferentes manejos com plantas de cobertura.

\begin{tabular}{ccccccc}
\hline Energia & & $\ldots \ldots \ldots \ldots \ldots \ldots \ldots \ldots \ldots$ & Indices de dispersão normalizados $\left(\mathrm{g} \mathrm{g}^{-1}\right) \ldots \ldots \ldots \ldots \ldots \ldots \ldots \ldots \ldots \ldots \ldots \ldots \ldots$ \\
$\mathrm{J} \mathrm{mL}$ & AF & AVER & AVNA & GR & SD & VE \\
\cline { 2 - 7 } 5,59 & $0,24 \mathrm{~b}$ & $0,29 \mathrm{~b}$ & $0,27 \mathrm{~b}$ & $0,18 \mathrm{c}$ & $0,54 \mathrm{a}$ & $0,33 \mathrm{~b}$ \\
16,7 & $0,41 \mathrm{c}$ & $0,63 \mathrm{~b}$ & $0,44 \mathrm{c}$ & $0,28 \mathrm{~d}$ & $0,90 \mathrm{a}$ & $0,65 \mathrm{~b}$ \\
22,3 & $0,51 \mathrm{~b}$ & $0,65 \mathrm{~b}$ & $0,58 \mathrm{~b}$ & $0,32 \mathrm{c}$ & $0,93 \mathrm{a}$ & $0,82 \mathrm{a}$ \\
33,5 & $0,54 \mathrm{~b}$ & $0,76 \mathrm{a}$ & $0,68 \mathrm{~b}$ & $0,42 \mathrm{c}$ & $1,00 \mathrm{a}$ & $0,87 \mathrm{a}$ \\
\hline
\end{tabular}

AF: amendoim Forrageiro, AVER: aveia + ervilhaca, AVNA: aveia + nabo, GR: Grama Pensacola, SD: solo descoberto, VE: vegetação espontânea. Médias seguidas de mesma letra na linha não diferem entre si pelo teste de Tukey $(p<0,05)$.

\section{Discussão}

Os índices de dispersão normalizados, obtidos por ultrassom, mostraram que a cobertura vegetal influenciou na estabilidade estrutural do solo em estudo. Mesmo nos níveis mais baixos de energia ultrassônica $\left(5,59 \mathrm{~J} \mathrm{~mL}^{-1}\right.$ e $\left.16,7 \mathrm{~J} \mathrm{~mL}^{-1}\right)$, desempenhos diferentes foram observados entre os tratamentos (Tabela 3, Figura 1). Para as condições deste estudo, o nível de energia específica aplicado de $16,7 \mathrm{~J} \mathrm{~mL}^{-1}$ foi o mais sensível para avaliar os efeitos das plantas de cobertura sobre a estabilidade dos agregados do solo.

Com o aumento da energia ultrassônica específica, menos diferenças entre os tratamentos foram encontradas (Tabela 3). Em níveis de energia mais elevados, a ausência de diferenças entre os tratamentos deve-se ao fato de os solos atingirem o platô de dispersão, que é limitado pelos níveis máximos de silte e argila (Silva et al., 2019).

A energia aplicada de $33,5 \mathrm{~J} \mathrm{~mL}^{-1}$ deixou o solo descoberto completamente disperso, não sendo uma boa escolha para a avaliação da estabilidade de agregados. Esse valor é muito inferior aos encontrados por Sá et al. (1999) para a dispersão total de Cambissolo $\left(33,5 \mathrm{~J} \mathrm{~mL}^{-1}<127 \mathrm{~J} \mathrm{~mL}^{-1}\right)$ demonstrando a vulnerabilidade do Argissolo estudado quando da ausência de cobertura vegetal.

Lehtinen et al. (2014) estudaram o padrão de desagregação de macroagregados de diferentes ordens de solo (Neossolo, Luvissolo, Argissolo e Cambissolo) em baixos níveis de energia ultrassônica (0-40 $\left.\mathrm{J} \mathrm{mL}^{-1}\right)$. Os autores constataram que apenas $2 \mathrm{~J} \mathrm{~mL}^{-1}$ foram suficientes para iniciar o processo de quebra dos agregados. Ribeiro, Lima, Mello, Sá e Oliveira (2009) constataram que a dispersão máxima dos agregados dos horizontes $\mathrm{Bi}$ e $\mathrm{C}$ de um Cambissolo Háplico foi alcançada com a aplicação de apenas 9,4 $\mathrm{J} \mathrm{mL}^{-1}$.

Macroagregados formados sob determinadas condições de manejo do solo podem sofrer ruptura e serem dispersos em níveis de energia ultrassônica muito baixos. Souza, Silva, Oliveira, Barbosa e Silva (2018) avaliaram com energia ultrassônica $\left(2,2-38,3 \mathrm{JmL}^{-1}\right)$ a macroagregação $(8,0-4,76 \mathrm{~mm})$ de um Argissolo Vermelho Amarelo distrófico cultivado com cafeeiros em diferentes sistemas de manejo implantados após prática de revolvimento do solo a $0,60 \mathrm{~m}$. Os autores demonstraram que o condicionamento químico realizado no solo, após prática de revolvimento profundo influenciou na reconstituição dos seus macroagregados. Macroagregados grandes e de maior estabilidade foram observados quando foi realizada adubação dos sulcos de plantio com organomineral e empregou-se à cobertura plástica nas linhas de cultivo dos cafeeiros. Todavia, quando foi realizada a adubação dos sulcos de plantio com fertilizantes químicos e o solo foi mantido descoberto, ou seja, sem a cobertura plástica na superfície do solo, a estabilidade dos macroagregados neoformados reduziu significativamente, com dispersão total em níveis de energia ultrassônica inferiores a $38,3 \mathrm{~J} \mathrm{~mL}^{-1}$.

Certamente, os macroagregados do manejo com fertilizantes químicos podem ter sido estabilizados por ligações mais fracas que aqueles que se formaram pela influência de adubação organomineral. Tem-se verificado a necessidade de aplicação de maiores níveis de energia ultrassônica para dispersar macroagregados formados em manejos conservacionistas de longa duração, sem revolvimento do solo e com aporte contínuo de matéria orgânica (Silva et al., 2016; Silva et al., 2020).

A maior estabilidade dos macroagregados de solo sob manejo com GR em relação ao solo sob o manejo com AF (Tabela 3) pode estar relacionada às diferenças nos aportes de COS (Tabela 2). Silva et al.(2016) e Souza et al.(2018) estudaram a desagregação de solos cultivados com cafeeiros utilizando sonificação e verificaram que nas camadas dos solos com maiores teores de COS foram necessários elevados níveis de energia ultrassônica para causar a desagregação e liberação dos macroagregados grandes $(8,0-4,76 \mathrm{~mm})$ em partículas discretas. 
Adicionalmente, macroagregação do solo também depende da qualidade e do grau de decomposição do resíduo que é adicionado ao solo pelas plantas de cobertura (Loss et al., 2015). Os resíduos de culturas mais facilmente degradáveis como o amendoim forrageiro, podem estar exercendo um efeito temporário na agregação do solo, uma vez que esses resíduos liberam compostos como polissacarídeos e açúcares, considerados agentes de agregação efêmeros (Zhu et al., 2017). Nestas condições, os agregados do solo sob AF seriam formados por agentes ligantes fracos (Silva et al., 2019), o que justificaria baixa estabilidade e maior dispersão com o aumento da energia ultrassônica aplicada.

Porém, resíduos com menor taxa de decomposição, como os de gramíneas (ex: Grama Pensacola), têm efeito gradativo e mais duradouro na agregação do solo devido à liberação de compostos de maior recalcitrância (lignina e fenóis) (Le Guillou, Angers, Maron, Leterme, Menasseri-Aubry, 2012). Consequentemente, no solo sob cultivo de GR, os agregados devem ser estabilizados por ligações fortes que exigem maiores níveis de energia ultrassônica para causar desagregação.

Corroborando com esta hipótese, Zhu et al.(2017) observou que os agregados dos solos incubados com resíduos vegetais de alfafa foram facilmente dispersos em níveis de energia ultrassônica inferiores aos aplicados em agregados de solo com cevada.

A utilização de plantas de cobertura do solo em monocultivo ou consorciadas e com diferentes sistemas radiculares foi eficiente em aumentar a agregação de um Latossolo Vermelho no Norte do Rio Grande do Sul (Pessotto et al., 2016). Da mesma forma, nesta pesquisa, o monocultivo do solo com AF e o uso de sistemas de rotação com AVER e AVNA trouxeram benefícios à estabilidade estrutural do solo refletida pelos valores de índices de dispersão normalizados inferiores aos do manejo do solo com VE e a testemunha SD.

Em diversos trabalhos tem-se mostrado que nos cultivos de gramíneas com leguminosas (AVER, AVNA) há um aumento da relação $\mathrm{C} / \mathrm{N}$ da fitomassa, o que promove a persistência dos resíduos culturais na superfície do solo (Aita \& Giacomini, 2003). Assim, esses sistemas de manejo conservacionistas podem proteger a superfície do solo dos agentes erosivos por longos períodos, o que também favorece a agregação do solo (Tabela 3).

Todavia, o curto período de cultivo das plantas de coberturas não foi suficiente para causar variações extremas nos teores de COS (Canalli et al., 2020). Mas, estudos com aveia, ervilhaca e nabo forrageiro em monocultivo mostraram que nestas plantas de cobertura, as maiores proporções de raízes finas ocorrem nas camadas superficiais $(0,0-0,10 \mathrm{~m})$, o que pode contribuir para o aumento do aporte de COS. Raízes finas devido a sua maior facilidade em penetrar e crescer no interior de agregados do solo tem sua decomposição dificultada (Redin et al., 2016).

Em trabalhos de pesquisa realizados com ervilhaca foram observadas produções de MS de raízes na camada de 0,0-0,20 m de profundidade do solo de $1.330 \mathrm{~kg} \mathrm{ha}^{-1}$, enquanto aveia preta produziu $1.850 \mathrm{~kg} \mathrm{ha}^{-1}$ (Redin et al., 2016). Nesse sentido, em longo prazo são esperados aumentos nos teores de COS do solo sob cultivo de AVER e AVNA, bem como incrementos em agregação, tendo em vista, que após 16 meses de experimento já foram observados benefícios na estabilidade estrutural do solo quando estes consórcios foram comparados ao SD e VE.

Em aspectos funcionais, para solos arenosos como o que está sendo estudado, o manejo conservacionista com plantas de cobertura, ao elevar o COS, pode proporcionar uma maior capacidade de

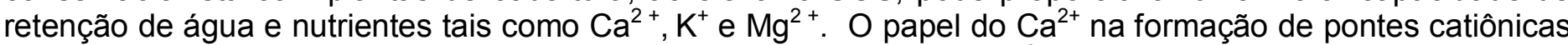
com argila e matéria orgânica também é conhecido. As interações $\mathrm{Ca}^{2+}$ - argila - matéria orgânica são vias ativas para a formação de microagregados, que são unidos por hifas de fungos e raízes que dão origem aos macroagregados (Briedis et al., 2012). Assim, os níveis mais baixos de $\mathrm{Ca}^{2+}$ podem ter contribuído para a maior susceptibilidade à desagregação do solo com ausência de cobertura vegetal (SD).

A variação sazonal da estabilidade estrutural do solo também desempenha um papel importante. Para a realização deste trabalho, apenas uma amostragem foi realizada em agosto de 2017 . No entanto, o padrão de agregação do solo pode ser influenciado por processos físicos relacionados às estações do ano, como precipitação, umidade e temperatura (Briedis et al., 2012). Dessa forma, em futuros estudos, os resultados observados neste trabalho poderão ser diferentes. Sugere-se um monitoramento temporal da agregação do solo nestes tratamentos para melhor compreensão dos processos atuantes (Canalli et al., 2020), a fim de demonstrar a variação cíclica que pode ser causada pelo manejo do solo em sua estabilidade estrutural.

Em longo prazo, ao contribuir para o aumento da estabilidade estrutural, sistemas de manejo com plantas de cobertura podem favorecer a proteção física da matéria orgânica, uma vez que esta é incorporada 
aos macroagregados do solo, ficando pouco exposta a processos microbianos, o que resultaria em menor taxa de mineralização e fluxo de $\mathrm{CO}_{2}$ para a atmosfera (Lal, 2015).

\section{Conclusões}

A energia ultrassônica específica de $16,7 \mathrm{JmL}^{-1}$ demonstrou maior sensibilidade para avaliação dos efeitos das diferentes coberturas vegetais sob a estabilidade estrutural do solo.

Após 16 meses da implantação do experimento, as plantas de cobertura GR, AF, AVNA e AVER já trouxeram benefícios à agregação do solo, sendo que no manejo com GR foram necessários níveis de energia ultrassônica maiores que $33,5 \mathrm{~J} \mathrm{~mL}^{-1}$ para causar a ruptura e dispersão total dos agregados.

Em curto prazo, as plantas de cobertura GR, AF, AVNA, AVER não causaram variações extremas nos teores de COS, indicando que estudos de longo prazo são necessários para se observar possíveis alterações nesse atributo do solo.

\section{Agradecimentos}

A Capes pela concessão de bolsas de estudo e ao Departamento de Ciência do Solo da Universidade Federal de Santa Maria pelo apoio incondicional as pesquisas.

\section{Referências}

Acosta, J.A.A., Amado, T.J.C., Silva, L.S., Santi, A., \& Weber, M.A. (2014). Decomposição da fitomassa de plantas de cobertura e liberação de nitrogênio em função da quantidade de resíduos aportada ao solo sob sistema plantio direto. Ciência Rural, 44, 801-809. http://dx.doi.org/10.1590/S0103-84782014005000002

Alvares, C. A., Stape, J.L., Sentelhas, P.C., de Moraes, J.L.G., Sparovek, G (2013). Köppen's climate classification map for Brazil. Meteorologische Zeitschrift, 22(6), 711-728. https://doi.org/10.1127/09412948/2013/0507

Aita, C., \& Giacomini, S.J. (2003). Decomposição e liberação de nitrogênio dos resíduos culturais de plantas de cobertura solteiras e consorciadas. Revista Brasileira de Ciência do Solo, 27, 601-612. http://dx.doi.org/10.1590/S0100-06832003000400004

Briedis, C., Moraes, J.C.S., Caires, E.F., Navarro, J.D.F., Inagaki, T.M., Boer, A., Neto, C.Q., Ferreira, A.D.O., Canalli, L.B., \& Santos, J.B. (2012). Soil organic matter pools and carbon-protection mechanisms in aggregate classes influenced by surface liming in a no-till system. Geoderma, 170, 80-88. https://doi.org/10.1016/j.geoderma.2011.10.011

Canalli, L. B. S., Santos, J. B., Benassi, D. A., Francisco, A. L. O., Benassi, C., Aguiar, A. N., Cordeiro, E., \& Mendes, R. S. (2020). Soil Carbon and Structural Quality in Crop Rotations under No-tillage System. Brazilian Archives of Biology and Technology, 63(spe), 1-12. https://doi.org/10.1590/1678-4324-solo-2020190603

Inagaki, T.M., Sá, J.C.M., Ferreira, A.O., Briedis, C., Tivet, F., \& Romaniw, J. (2016). Macroagregados como indicadores de qualidade em sistema plantio direto. Revista Plantio Direto, 151 (5), 2-8.

Embrapa. Empresa Brasileira de Pesquisa Agropecuária. Centro Nacional de Pesquisas de Solos. Manual de métodos de análises de solos. 2.ed. Rio de Janeiro: Embrapa Solos, 2011. 230p.

Lal, R. (2015). Soil carbon sequestration and aggregation by cover cropping. Journal Soil Water Conservation, 70, 329-339. https://doi.org/10.2489/jswc.70.6.329

Le Guillou, C., Angers, D.A., Maron, P.A., Leterme, P., \& Menasseri-Aubry, S. (2012). Linking microbial community to soil water-stable aggregation during crop residue decomposition. Soil Biology and Biochemistry, 50,126-133. https://doi.org/10.1016/j.soilbio.2012.03.009

Lehtinen, T., Lair, G.J., Mentler, A., Gísladóttir, G., Ragnarsdóttir, K.V., \& Blum, W.E.H. (2014). Soil Aggregate Stability in Different Soil Orders Quantified by Low Dispersive Ultrasonic Energy Levels. Soil Science Society American Journal, 78, 713-723. https://doi:10.2136/sssaj2013.02.0073

Loss, A., Basso, A., Oliveira, B.S., Koucher, L.P., Oliveira, R.A., Kurtz, C., Lovato, P.E., Curmi, P., Brunetto, G., \& Comin, J.J. (2015). Carbono orgânico total e agregação do solo em sistema de plantio direto 
agroecológico e convencional de cebola. Revista Brasileira de Ciência do Solo, 39,1212-1224. https://doi.org/10.1590/01000683rbcs20140718

Loss, A., Pereira, M. G., Anjos, L. H. C., Giacomo, S. G., \& Perin, A.(2011). Agregação, carbono e nitrogênio em agregados do solo sob plantio direto com integração lavoura-pecuária. Pesquisa Agropecuária Brasileira, 46(10), 568-76. http://dx.doi.org/10.1590/S0100-204X2011001000022

Pessotto, P.P., Silva, V.R., Ortigara, C., Koppe, E., Strojaki, T., \& Santi, A.L.(2016). Influência de diferentes plantas de cobertura nas propriedades físicas de um Latossolo vermelho. Revista Agrarian, 9 (34), 348-356. http://ojs.ufgd.edu.br/index.php/agrarian/article/view/4519/3682

R Development Core Team. (2018). R: a language and environment for statistical computing. R Foundation for Statistical Computing, Vienna, Austria.

Redin, M., Giacomini, S. J., Ferreira, P. A. A., \& Eckhardt, D. P. (2016). Plantas de cobertura de solo e agricultura sustentável: espécies, matéria seca e ciclagem de carbono e nitrogênio. In: Tiecher, T. Manejo e conservação do solo e da água em pequenas propriedades rurais no sul do Brasil: práticas alternativas de manejo visando a conservação do solo e da água. Porto Alegre: UFRGS, p. 7-22, 2016.

Ribeiro, B.T., Lima, J.M., Oliveira, G.C., Curi, N., Silva, E.A., \& Silva, B.M. (2017). Ultrasonic aggregate breakdown of an Oxisol as affected by cavitation intensity. Communications in Soil Science and Plant Analysis, 48, 818-824. http://dx.doi.org/10.1080/00103624.2017.1299170

Ribeiro, B.T., Lima, J.M., Curi, N., \& Oliveira, G.C. (2013). Aggregate breakdown and dispersion of soil samples amended with sugarcane vinasse. Scientia Agricola, 70, 435-41. http://dx.doi.org/10.1590/S010390162013000600009

Ribeiro, B.T., Lima, J.M., Mello, C.R., Sá, M.A.C., \& Oliveira, G.C. (2009). Relationship between raindrops and ultrasonic energy on the disruption of a Haplic Cambisol. Ciência e Agrotecnologia, 33, 814-823. http://dx.doi.org/10.1590/S1413-70542009000300021

Sá, M.A.C., Lima, J.M., Silva, M.L.N., \& Dias Júnior, M.S. (1999). Índices de desagregação do solo baseado em energia ultra-sônica. Revista Brasileira de Ciência do Solo, 23, 525-531. http://dx.doi.org/10.1590/S010006831999000300005

Santos, H.G., Jacomine, P.K.T., Anjos, L.H.C., Oliveira, V.A., Lumbreras, J.F., Coelho, M.R., Almeida, J.A., Araújo Filho, J.C., Oliveira, J.B., \& Cunha, T.J.F. (2018). Sistema Brasileiro de Classificação de Solos. $5^{\mathrm{a}}$ ed., rev. e ampl. Brasília, DF: Embrapa. 356p.

Schomakers, J., Mentler, A., Steurer, T., Klik, A., \& Mayer, H. (2011). Characterization of soil aggregate stability using low intensity ultrasonic vibrations. International Agrophysics, 25,165-72.

Silva, É.A., Benevenute, P.A.N., Oliveira, G.C., Zinn, Y.L., Silva, B.M., Melo, L.B.B., Reis, T.H.P., Oliveira, C.H.C.,Guimarães, P.T.G., \& Carducci, C.E (2020). Soils under Plastic and Grass Cover: Effects on Soil Aggregation and Nutrient Cycling in Brazilian Coffee Growing. Agricultural Research \& Technology: Open Access Journal, 24(2): 74-81. http://dx.doi.org/10.19080/ARTOAJ.2020.25.556265

Silva, E.A., Reinert, D.J., Reichert, J.M., Mallmann, M.S., Pereira, M.A., Pons, S.S., \& Foggiato, W.S. (2019). Soil conservation management with cover crops: effects on critical energy levels, release and dispersion of aggregates. Bragantia, 78, 444-453. http://dx.doi.org/10.1590/1678-4499.20180323

Silva, É.A., Oliveira, G.C., Carducci, C.E., Lima, J.M., Melo, L.B.B., \& Benevenute, P.A.N. (2016). Stability of soil aggregates in Latosols and Cambisols via standard method and sonification. African Journal of Agricultural Research, 11, 3894-3903. https://doi.org/10.5897/AJAR2016.11192

Stefanoski, D. C., Santos, G. G., Marchão, R. L., Petter, F. A., \& Pacheco, L. P.(2013). Uso e manejo do solo e seus impactos sobre a qualidade física. Revista Brasileira de Engenharia Agrícola e Ambiental, 17(12),1301-1309. https://doi.org/10.1590/S1415-43662013001200008

Souza, L.A., Silva, E.A., Oliveira, G.C., Barbosa, S.M., \& Silva, B.M (2018). Análise qualitativa e quantitativa de agregados de solo sob filme plástico associado à fertilização organomineral em área cafeeira. Scientia agraria, 19, 142-153. https://doi. 10.5380/rsa.v19i2.52826 


\section{-igarARIAN}

Tedesco, M.J., Gianello, C., Bissani, C.A., Bohnen, H., \& Volkweiss, S.J.(1995). Analises de solo, plantas e outros materiais. Universidade Federal do Rio Grande do Sul, Porto Alegre, RS, Brazil.

Tivet, F., Moraes, J.C.S., Lal, R., Briedis, C., Borszowskei, P.R., Santos, J.B., Farias, A., Eurich, G., Hartman, D.C., Junior, M.N., Bouzinac, S., \& Séguy, L. (2013). Aggregate C depletion by plowing and its restoration by diverse biomass-C inputs under no-till in sub-tropical and tropical regions of Brazil. Soil Tillage Research, 126, 203-218. https://doi.org/10.1016/j.still.2012.09.004

Torres, J.L.R., Pereira, M.G., Assis, R.L., \& Souza, Z.M. (2015). Atributos físicos de um latossolo vermelho cultivado com plantas de cobertura, em semeadura direta. Revista Brasileira de Ciência do Solo, 39, 428-437. http://dx.doi.org/10.1590/01000683rbcs20140597

Zhu, Z., Angers, D. A., Field, D. J. and \& Minasny, B. (2017). Using ultrasonic energy to elucidate the effects of decomposing plant residues on soil aggregation. Soil and Tillage Research, 167, 1-8. https://doi.org/10.1016/j.still.2016.10.002 